\title{
Genetic and pathogenic diversity of severe fever with thrombocytopenia syndrome virus (SFTSV) in South Korea
}

\author{
Seok-Min Yun, ${ }^{1,2}$ Su-Jin Park, ${ }^{1,3}$ Young-II Kim, ${ }^{1,3}$ Sun-Whan Park, ${ }^{4}$ Min-Ah Yu, ${ }^{1,3}$ Hyeok-II Kwon, ${ }^{1,3}$ \\ Eun-Ha Kim, ${ }^{1,3}$ Kwang-Min Yu, ${ }^{1,3}$ Hye Won Jeong, ${ }^{1,5}$ Jungsang Ryou, ${ }^{2}$ Won-Ja Lee, ${ }^{2}$ Youngmee Jee, ${ }^{6}$ \\ Joo-Yeon Lee, ${ }^{2}$ and Young Ki Choi ${ }^{1,3}$ \\ 'Department of Microbiology, College of Medicine and Medical Research Institute, Chungbuk National University, \\ Cheongju, Chungcheongbuk-do, South Korea. 'Division of Emerging Infectious Disease and Vector Research, Center for \\ Infectious Diseases Research, National Institute of Health, Korea Centers for Disease Control and Prevention, Cheongju, \\ Chungcheongbuk-do, South Korea. ${ }^{3}$ Zoonotic Infectious Diseases Research Center, Chungbuk National University, \\ Cheongju, Chungcheongbuk-do, South Korea. ${ }^{4}$ Jeju National Quarantine Station, Centers for Disease Control and \\ Prevention of Korea, Jeju, Jeju-do, South Korea. ${ }^{5}$ Department of Internal Medicine, Chungbuk National University College \\ of Medicine, Cheongju, Chungcheongbuk-do, South Korea. ${ }^{6}$ Center for Infectious Diseases Research, National Institute of \\ Health, Korea Centers for Disease Control and Prevention, Cheongju, Chungcheongbuk-do, South Korea.
}

To investigate nationwide severe fever with thrombocytopenia syndrome virus (SFTSV) infection status, we isolated SFTSVs from patients with suspected severe fever with thrombocytopenia syndrome (SFTS) in 207 hospitals throughout South Korea between 2013 and April 2017. A total of 116 SFTSVs were isolated from 3137 SFTS-suspected patients, with an overall $21.6 \%$ case fatality rate. Genetic characterization revealed that at least 6 genotypes of SFTSVs were co-circulating in South Korea, with multiple reassortments among them. Of these, the genotype B-2 strains were the most prevalent, followed by the $A$ and $F$ genotypes. Clinical and epidemiologic investigations revealed that genotype $B$ strains were associated with the highest case fatality rate, while genotype A caused only one fatality among 10 patients. Further, ferret infection studies demonstrated varying clinical manifestations and case mortality rates with different strains of SFTSV, which suggests this virus could exhibit genotype-dependent pathogenicity.

Authorship note: SMY, SIP, and YIK contributed equally to this work as co-first authors. JYL and YKC are cosenior authors

Conflict of interest: The authors have declared that no conflict of interest exists.

Copyright: (c) 2020, American Society for Clinical Investigation.

Submitted: April 12, 2019 Accepted: December 10, 2019 Published: January 30, 2020.

Reference information: /CI Insight. 2020;5(2):e129531.

https://doi.org/10.1172/jici.

insight.129531.

\section{Introduction}

Severe fever with thrombocytopenia syndrome (SFTS) - an emerging tick-borne infectious disease with a high fatality rate and symptoms including severe fever and thrombocytopenia - was first reported in China in $2011(1,2)$. The causative agent, the SFTS virus (SFTSV), was subsequently identified in South Korea and Japan in 2013 following lethal infections in humans (3, 4). SFTSV (now renamed Huaiyangshan banyangvirus) belongs to the genus Banyangvirus in the family Phenuiviridae $(5,6)$ along with other novel tick-borne Banyangviruses, including Heartland virus and Guertu virus $(5,7,8)$. The genome of SFTSV has negative-stranded RNA segments, including the $L$ segment, which encodes the RNA-dependent RNA polymerase (RdRp); the $M$ segment, which encodes the surface glycoproteins $\mathrm{Gn}$ and Gc; and the $S$ segment, which harbors a nucleoprotein (NP); and a nonstructural $S$ segment (NS) protein, which is encoded via an ambisense strategy $(2,9)$. Since the first report of SFTSV in humans in 2011 (2), the number of human cases has rapidly increased each year in China, South Korea, and Japan (10-12). Although the average case fatality rate varies among regions and years (13), the mean mortality rate of SFTS cases has remained relatively high in Japan (27\%), South Korea (23.3\%), and China (5.3\%-16.2\%) (10-12). Further, SFTSV infection has clinical features including high fever, thrombocytopenia, and leukocytopenia and can cause central nervous system manifestations including headache, confusion, and seizure (14-16). The reason for the difference in mortality rates between countries and the mechanisms underlying the varied clinical manifestations caused by this infection are largely unknown; however, underlying disease conditions are suspected $(17,18)$. Recently, several studies reported the identification of at least 6 different genotypes of SFTSV in East Asian countries and that the prevalence of each genotype varied by country (19-21). 
To understand the differing clinical manifestations of SFTSV infections, several studies were analyzed to compare host factors such as age, cytokine profile in sera, proportions of PBMCs, and viral titers in the central nervous system (22-25). However, whether the pathogenic potential of different SFTSV strains varies remains unclear. To this end, we characterized a total of 116 Korean SFTSVs isolates and investigated the epidemiological and clinical records of patients, including age and clinical outcomes. Further, we evaluated the pathogenic characteristics of each SFTSV genotype using an animal model.

\section{Results}

Characteristics of SFTS in human patients. A total of 3137 serum samples were collected from hospitalized patients with suspected SFTS between 2013 and April 2017. These patients experienced symptoms of SFTS, such as high fever $\left(\geq 38^{\circ} \mathrm{C}\right)$, vomiting, diarrhea, fatigue, thrombocytopenia, leukocytopenia, and spots resulting from tick bites, and were treated at 207 hospitals throughout South Korea. Specimens from 342 of these patients were found to be positive for SFTSV by real-time reverse-transcription PCR, and there were $74(21.6 \%)$ confirmed fatalities (Figure 1A). Of the 342 SFTSV-positive cases, a total of 116 human-derived SFTSVs were isolated in Vero E6 cells. Of these, 38 (32.8\%) viruses were obtained from fatal cases and 26 were isolated from specimens taken from farmers ( 26 of 116, 22.4\%). The detailed regional distribution of SFTS cases analyzed in this study is shown in Supplemental Figure 1; supplemental material available online with this article; https://doi.org/10.1172/jci.insight.129531DS1.

The most frequent symptoms of SFTS were high fever $\left(\geq 38^{\circ} \mathrm{C}\right)$, gastrointestinal symptoms (vomiting, diarrhea, and nausea), and thrombocytopenia and/or leukocytopenia (Supplemental Table 1). Patient age ranged from 19 to 89 years, with a mean of 66.6 , and the majority of virus-isolated cases were patients aged $\geq 50$ years (Figure $1, \mathrm{~B}$ and C). Moreover, those $\geq 70$ years ( 46 of $74,62.2 \%$ ) showed the highest mortality rate $(P<0.05)$, followed by patients $60-69$ years $(22$ of $74,29.7 \%)$ and $50-59$ years $(6$ of $74,8.1 \%)$, suggesting age is an important factor for SFTSV-induced fatality (Figure 1C). There were no sex differences in infection or case fatality rates (Figure 1D).

Genetic and phylogenetic analyses. To investigate the genetic characteristics of SFTSVs, full genomic sequences of all 116 Korean SFTSVs were analyzed using the next-generation sequencing (NGS) deep sequencing method. These sequences, as well as all known SFTSV full-length sequences available in GenBank, were subjected to phylogenetic analysis (Figure 2 and Supplemental Figure 2). The results show that Korean SFTSVs can be roughly classified into A-F genotypes based on the classification method previously used in China and Korea $(19,26)$. The majority of Korean SFTSV isolates ( $n=92$ of $133,69.2 \%$ ) were classified as the B genotype. However, it is noteworthy that repeated analysis of genotype B strains resulted in their subdivision into 3 different genotypes, B-1, B-2, and B-3, with strong bootstrap values (>70) (Figure 2). In addition, the $L$ and $M$ segments of the $16 M S 373$ strain, as well as the $L$ segments of the $16 K S 77$ and KADGH4 strains, did not cluster with known A-F genotype groups, although their internal $M$ and/or $S$ segments clustered with genotype B-1, B-2, or B-3 (Figure 2). Further, the $L$ segment of the ZJ2013-06 Chinese isolate and 3 strains of Japanese origin (SPL057A, SPL097A, and SPL100A) also aligned with unclassified clades (Figure 2A).

Genotypes of Korean SFTSVs. Based on the phylogenetic analyses, the Korean SFTSVs were differentiated into 6 pure genotype groups (A-F) and their reassortant genotype groups (Figure 3). In the A-F pure genotype groups, the most prevalent genotype was B-2 $(n=48,36.1 \%)$, followed by B-3 $(n=28,21.1 \%)$, and B-1 $(n=16$, $12.0 \%$ ) (Figure 3A). Although the first Korean SFTSV strain identified was genotype $\mathrm{F}$ in 2012, its prevalence was lower than that of the B (B-1, B-2, and B-3) and A genotypes (Figure 4A). Further, while genotype D viruses ( $n=5,3.8 \%$ ) were detected in 2013 and 2014, in recent years this genotype has rarely been detected in Korea. In contrast, genotype $\mathrm{C}$ and E SFTSV strains were not detected during this study (Figure 3A). The most prevalent genotype in Japan was B-2, and the overall genotype distribution was similar to that seen in South Korea, although genotypes $\mathrm{A}$ and $\mathrm{F}$ were not reported (Supplemental Table 2). In contrast, the Chinese SFTSV strains were diverse, with 14 genotypes reported, the most prevalent being genotype $\mathrm{F}$.

In reassortant groups, R-3 ( $n=6)$, which results from recombination between the B-1 and B-3 genotypes, was the most common, followed by R-2, which results from recombination between the B-1 and B-2 genotypes $(n=4)$ (Figure $3 \mathrm{~B})$. Further, even in a single strain various reassortants were present, such as R-1 (B-1 and B-2), R-4 (B-3 and F), R-5 (C and D), and R-6 (B-1 and D) (Figure 3B). The R-7 virus had an unclassified $L$ gene and $M$ and $S$ genes from the B-2 and B-1 genotypes, respectively. In addition, the R- 8 virus also has the unclassified $L$ gene, while the $M$ and $S$ segments of this strain originated from B-3 and B-1 genotypes, respectively. Further, the R-9 virus also contained unclassified $L$ and $M$ segments, while the $S$ segment originated 
A

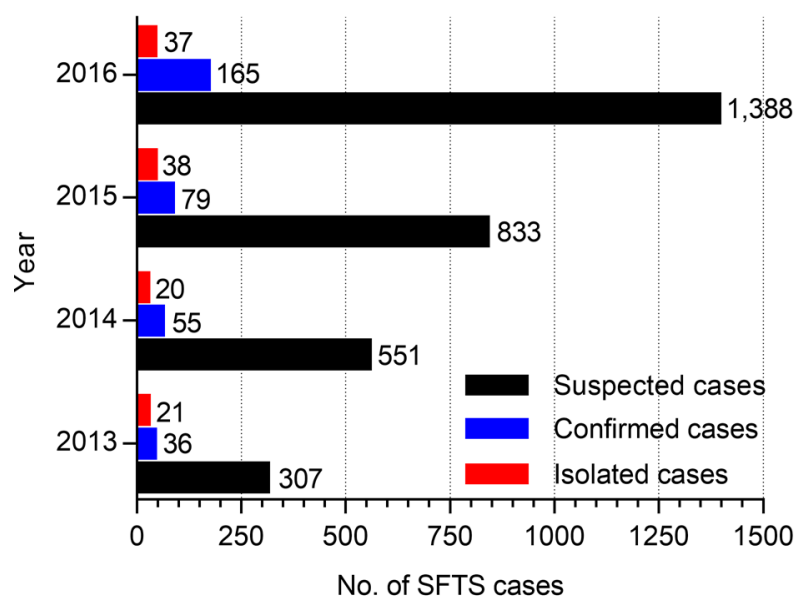

C

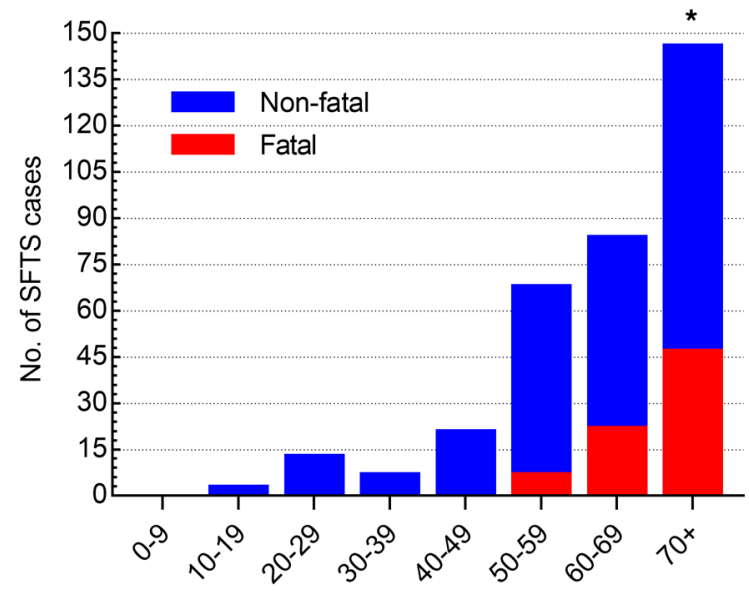

Age group (years)
B

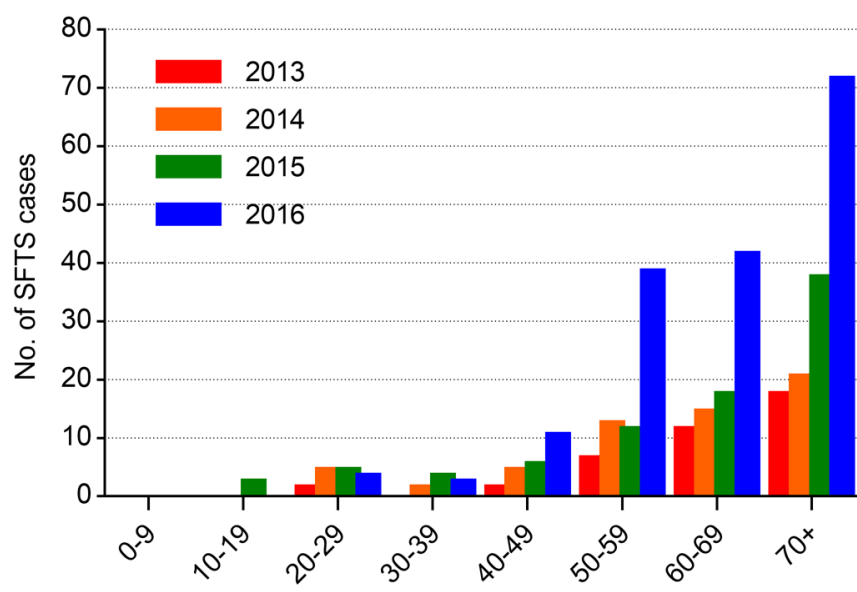

Age group (years)

D

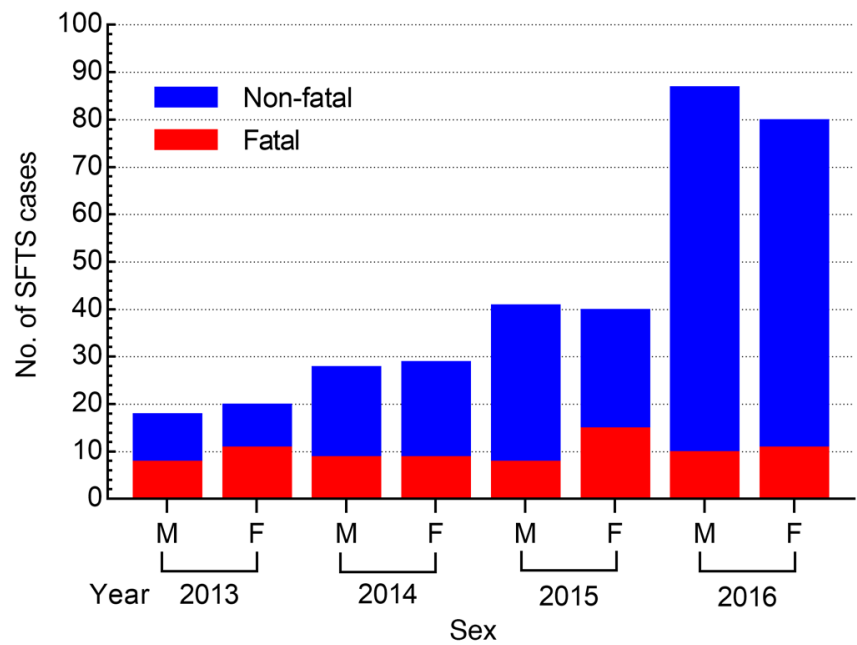

Figure 1. Distribution of SFTS cases between 2013 and 2016. (A) Suspected, confirmed, and isolated human cases of SFTS in South Korea from 2013 to 2016. (B) Age distribution of confirmed SFTS cases. (C) Number of SFTS fatalities in confirmed cases. (D) Ratio of nonfatal to fatal cases by sex and year. M, male; F, female. Asterisks indicate statistically significant differences in mortality rates between each age group of infected patients as determined by 2-tailed Mantel-Cox or Mann-Whitney $U$ test $\left({ }^{*} P<0.05\right)$.

from genotype B-1 (Figure 3B). These results suggest that Korean SFTSV strains were actively undergoing evolution through dynamic reassortments resulting in the creation of various novel genotypes.

Association between the case fatality rate and SFTSV genotype. To investigate whether different genetic phenotypes induced different case fatalities rates, we first analyzed the human mortality rate across genotypes (Figure 4). Most genotypes of SFTSV were detected annually starting in 2013, but the reassortants (R-1, R-2, R-5, R-8, and R-9) were first detected in 2016 with low incidence (Figure 4A). Although most genotypes of SFTSVs were associated with fatalities, the B-2 genotype showed the highest incidence (48 of 133 cases) and a significantly higher mortality rate (21 of 48 patients, $43.8 \%)$ than the other genotypes $(P$ $<0.05$ ) (Figure 4B). The F genotype also showed a high mortality rate (4 of 9 cases, $44.4 \%$ ), although the incidence rate was lower than that of B-3 ( 8 of 28 cases) and B-1 (3 of 16 cases) genotypes. In addition, the A genotype showed the lowest mortality rate ( 1 of 10 cases) compared with the other genotypes (Figure 4B). Of the reassortant genotypes, R-3 showed the strongest correlation with high mortality (4 of 6 cases, $66.7 \%$ mortality rate), followed by the R-2 genotype ( 2 of 4 cases, $50 \%$ mortality). Although only single cases of each of the other novel R genotypes were detected in this study, it should be noted that genotype R-6, R-7, and R-9 viruses were isolated from fatal cases of SFTSV infection. Further, there was a clear association between the case mortality rate and age of infected patients as revealed by analysis of clinical 

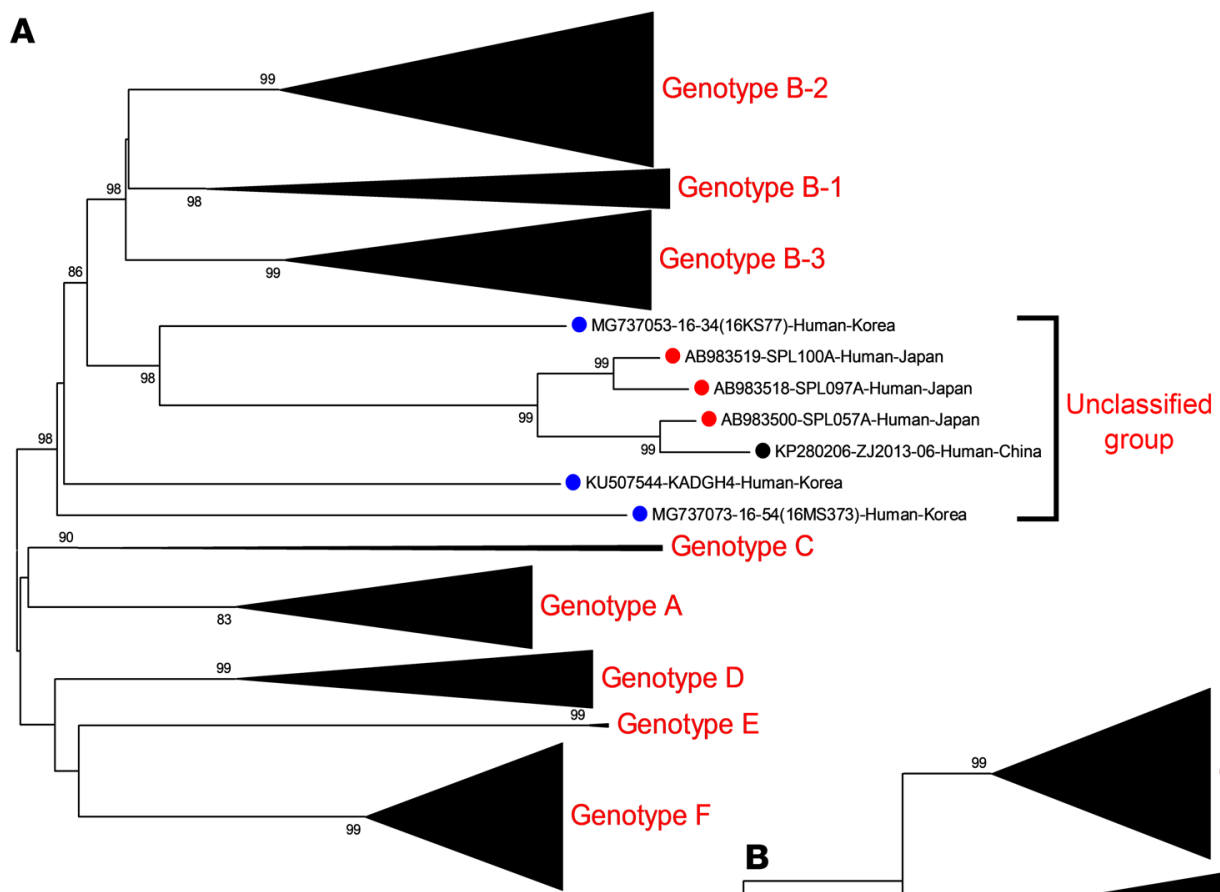
Genotype C
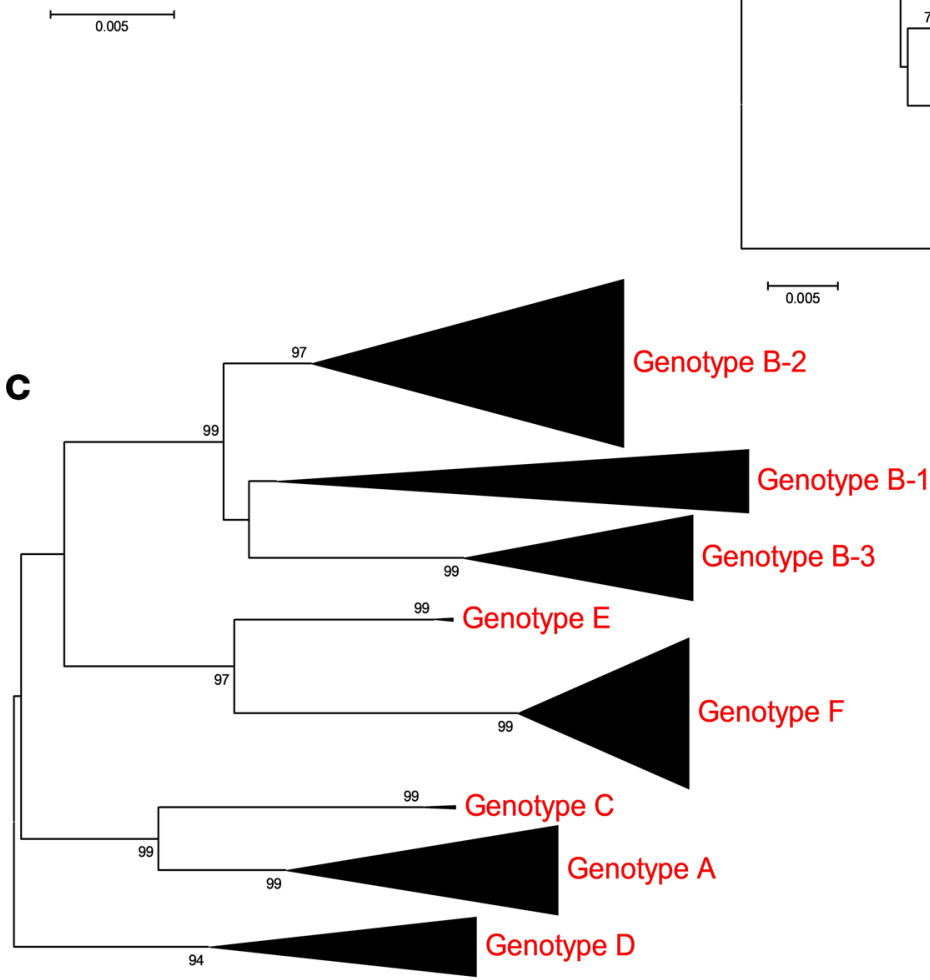

Figure 2. Phylogenetic analysis based on the complete ORF sequences of $L, M$, and $S$ gene segments of Korean SFTSVs compared with those from China and Japan. (A) L segment. (B) M segment. (C) $S$ segment. Compressed versions of the ML trees based on the Kimura 2-parameter model were constructed and tested by bootstrap analysis with 1000 replications. The scale bars $(0.005)$ indicate the number of nucleotide substitutions per site, and the phylogenetic branches were supported with greater than $70 \%$ bootstrap values. The SFTSV strains from China, Japan, and South Korea are marked with black, red, and blue closed circles, respectively. The full versions of the ML trees in this study are shown in Supplemental Figure 2.

records of confirmed SFTSV cases (Figure 4C). Most fatalities were in patients older than 60 (42 of 47 cases, 89.4\%), although 5 fatalities $(10.6 \%)$ occurred in patients aged $50-59(P<0.05)$.

Genotype-specific pathogenicity of SFTSVs. To confirm that different genotypes of SFTSVs exhibit different clinical manifestations, each randomly selected SFTSV strain (A [CB2], B-1 [CB3], B-2 [CB7], B-3 [CB6], D [CB8], F [16KS89], R-1 [16MS299], R-2 [16MS310], R-3 [CB1], R-4 [KACNH2], R-5 [16MS322], R-6 [16KS55], R-7 [KADGH4], R-8 [16KS77], R-9 [16MS373]) was inoculated into young adult and aged ferrets, a proven SFTSV infection model (27-29), and clinical symptoms, hematology, and mortality were monitored for 14 


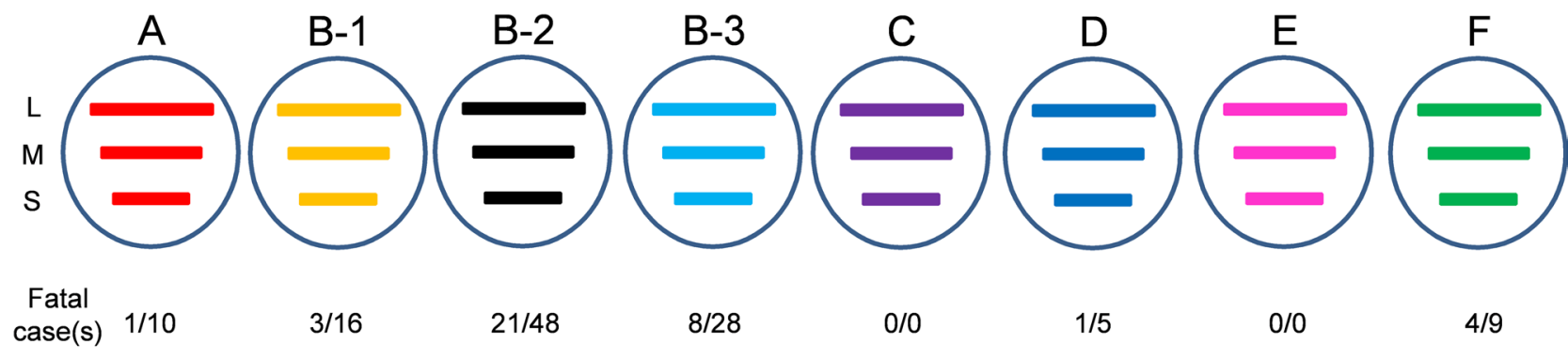

B Reassortant group

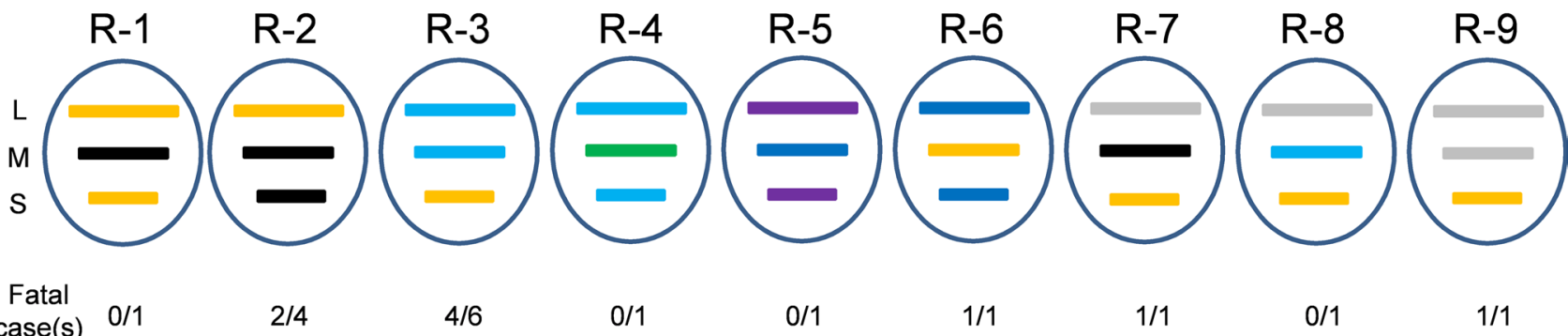

Figure 3. Genotypes of Korean SFTSVs isolated from human sera. The SFTSVs were assigned to different genotypes based on the genetic origin of each segment as determined by phylogenic analysis. The SFTSVs were differentiated into at least 15 genotypes, including genetic reassortant viruses. Gene segments from top to bottom are $L, M$, and S. The bars indicate pure groups of genotypes (A) A (red), B-1 (yellow), B-2 (black), B-3 (light blue), C (purple), D (blue), E (pink), F (green); and reassortant groups of genotypes (B) R-1 to R-9, including the unique clustering genotype (gray).

days. To optimize the infection dose in ferrets, we analyzed the growth property of each selected virus in Vero E6 cells (Supplemental Table 3). The B-1 virus showed the most efficient replication compared with the other viruses ( $10^{7.6}$ focus-forming units $[\mathrm{FFU}] / \mathrm{mL}$ ), and the B-2, B-3, R-1, R-2, R-3, R-4, and R-6 viruses exhibited peak titers of approximately $10^{6.6}$ to $10^{7.3} \mathrm{FFU} / \mathrm{mL}$. The genotype A, D, F, R-5, R-7, R-8, and R-9 strains showed attenuated viral titers in cells $\left(10^{5.3}\right.$ to $\left.10^{5.9} \mathrm{FFU} / \mathrm{mL}\right)$ compared with the other genotypes (Supplemental Table 3). However, all viruses tested in this study grew up to at least $10^{5.0} \mathrm{FFU} / \mathrm{mL}$ at peak titers; hence, we determined $10^{5.0} \mathrm{FFU} / \mathrm{mL}$ to be the optimal infection dose for the ferrets.

In this study, none of the young adult ferrets infected with any of the genotypes died or experienced weight loss (Supplemental Figures 3 and 4). Further, most young adult ferrets showed only mildly elevated body temperatures from 4 to 6 days post-infection (dpi) and short periods of viremia (within 10 dpi) compared with aged ferrets (Figure 5, Figure 6, and Supplemental Figures 3, 4, and 5). In contrast, in aged ferrets, pure genotypes B (B-1, B-2, and B-3) and D caused 100\% mortality within 12 dpi, with high fever, significant body weight loss (more than 20\%), and high virus RNA copy numbers in collected blood samples (greater than 4 $\log _{10} / \mathrm{mL}$ at 8-10 dpi) (Figure 5, B and C; Figure 6, A and B; and Supplemental Figure 3, B-E). Genotype A- and F-infected aged ferrets showed 40\% (2 of 5) and 60\% (3 of 5) mortality, respectively (Figure $5 \mathrm{~A}$ and Figure 6C). Further, viral RNAs were detected until 12 dpi in surviving ferrets infected with genotype A and $\mathrm{F}$ virus, although their body weight and temperature recovered to the normal range (Supplemental Figure 3, A and F). In the reassortant genotype groups, R-2, R-3, and R-5 caused 100\% mortality, with high fever and body weight loss (more than 20\%) (Supplemental Figure 4, B, C and E, and Supplemental Figure 5, B, C, and E). Further, these groups showed much higher peak viral RNA copy numbers $\left(>4.0 \log _{10} / \mathrm{mL}\right)$ in sera compared with the other reassortant groups $\left(<4.0 \log _{10} / \mathrm{mL}\right.$ ) (Supplemental Figure 5 , A-I). In aged ferrets the R-1, R-4, R-6, and R-7 viruses caused 80\% (4 of 5), 60\% (3 of 5), 40\% (2 of 5), and 40\% (2 of 5) mortality (Supplemental Figure 5, A, D, F, and G), while R-8 and R-9 caused only 20\% (1 of 5) mortality (Supplemental Figure 5, H and I), with mildly elevated body temperatures and viral RNA copy numbers $\left(<4.0 \log _{10} / \mathrm{mL}\right)$ (Supplemental Figure 4, H and I, and Supplemental Figure 5, H and I). 
A

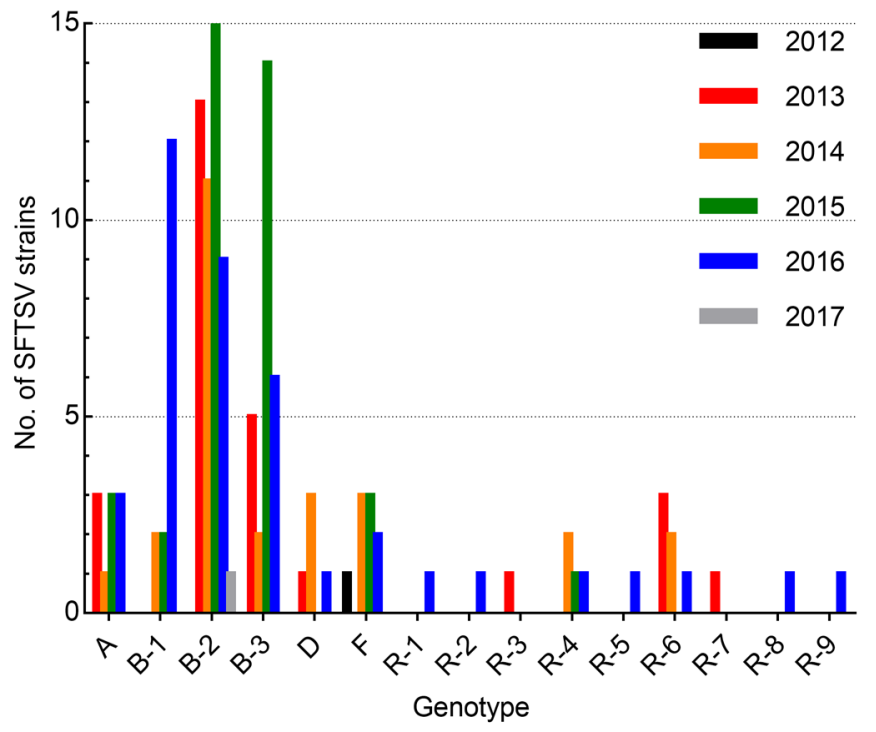

C

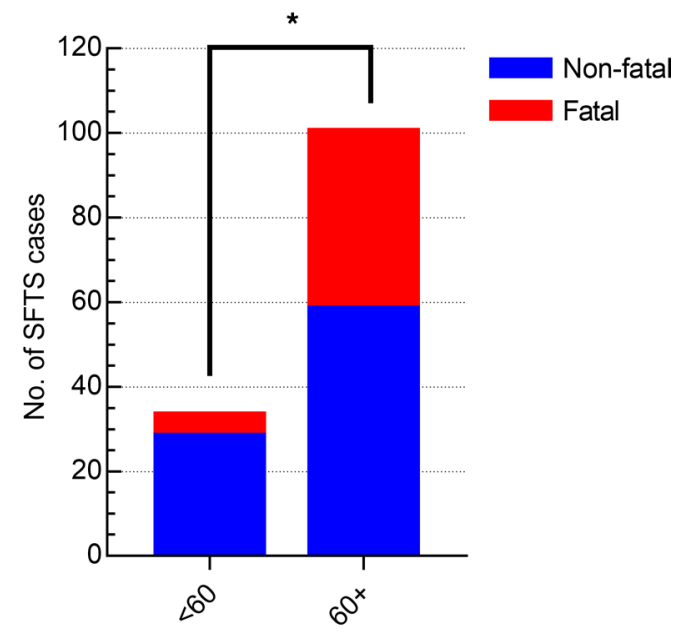

Age group (years)

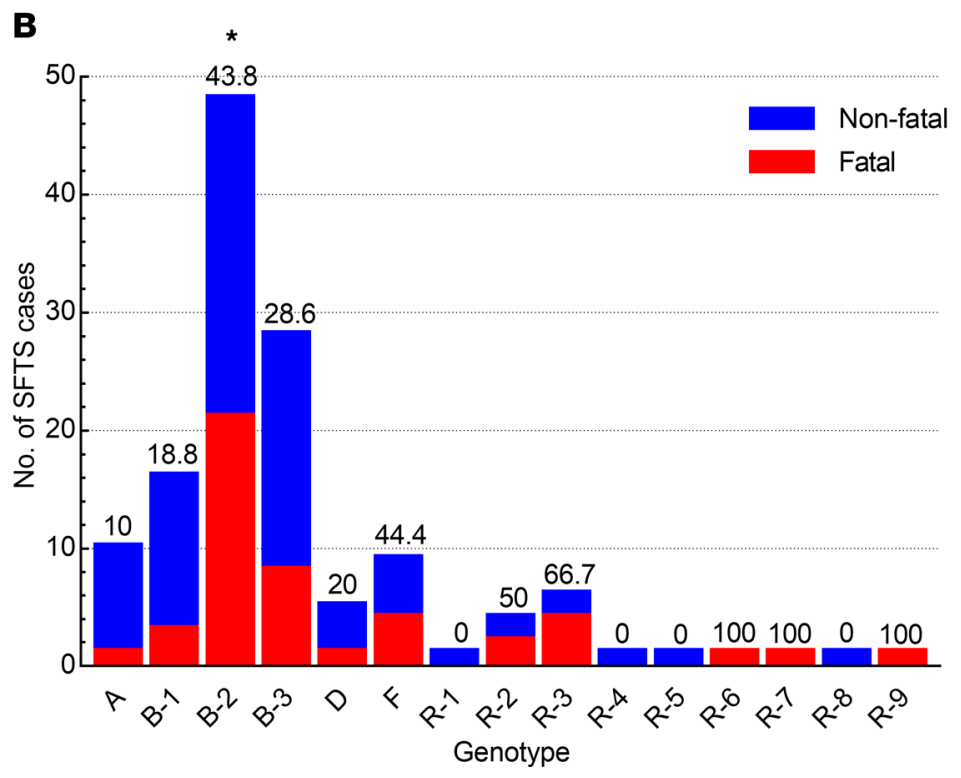

Figure 4. Prevalence and fatality rates of each genotype by year. (A) Incidence of each genotype of SFTSV from 2012 to 2017. (B) Ratio of nonfatal to fatal cases for each genotype. The case fatality percentage (\%) is shown at the top of each bar. (C) Ratio of nonfatal to fatal cases by age group. Asterisks indicate statistically significant differences in mortality rates between each genotype (B) or aged group (C) determined by Fisher's exact test $\left({ }^{*} P<0.05\right)$.

Hematological analysis revealed that most pure and reassortant genotype-infected young adult ferrets maintained normal platelet numbers (more than $300 \times 10^{3} / \mu \mathrm{L}$ ), although a single B-1 and a single $\mathrm{D}$ genotype-infected ferret showed decreased platelet numbers at 4 and $8 \mathrm{dpi}$, respectively (Figure 5B and Figure 6B). Interestingly, all fatal cases exhibited persistent severe thrombocytopenia (more than 4 days below $100 \times 10^{3} / \mu \mathrm{L}$ ), while surviving animals recovered their platelet numbers within 12 days even in aged ferrets (Figure 5, Figure 6, and Supplemental Figure 5). In young adult ferrets, WBC counts slightly decreased at 4 to 6 dpi compared with the initial day of infection but remained in the normal range $\left(2.5 \times 10^{6}\right.$ to $\left.16.7 \times 10^{6} / \mu \mathrm{L}\right)$. Further, the ALT and AST levels were slightly increased until 8 dpi, but returned to the normal range (ALT normal range: 49-242.8 UI ${ }^{-1}$; AST normal range: 40.1-142.7 UI-1) after 10 dpi (Supplemental Figures 6 and 7). However, in aged ferrets, regardless of the genotype, most SFTSV-infected ferrets exhibited rapid thrombocytopenia, decreased WBC counts, and increased AST/ALT levels (from 4 to $10 \mathrm{dpi}$ ), although surviving ferrets had recovered to the normal range of each parameter at 14 dpi (Supplemental Figures 6 and 7). Taken together, these results demonstrate that both the age of the infected animal and the SFTSV genotype are associated with varied clinical outputs, suggesting the genotype-specific pathogenic potential of this virus. 
O Young adult ferrets

$\checkmark$ Aged ferrets

Survival rate
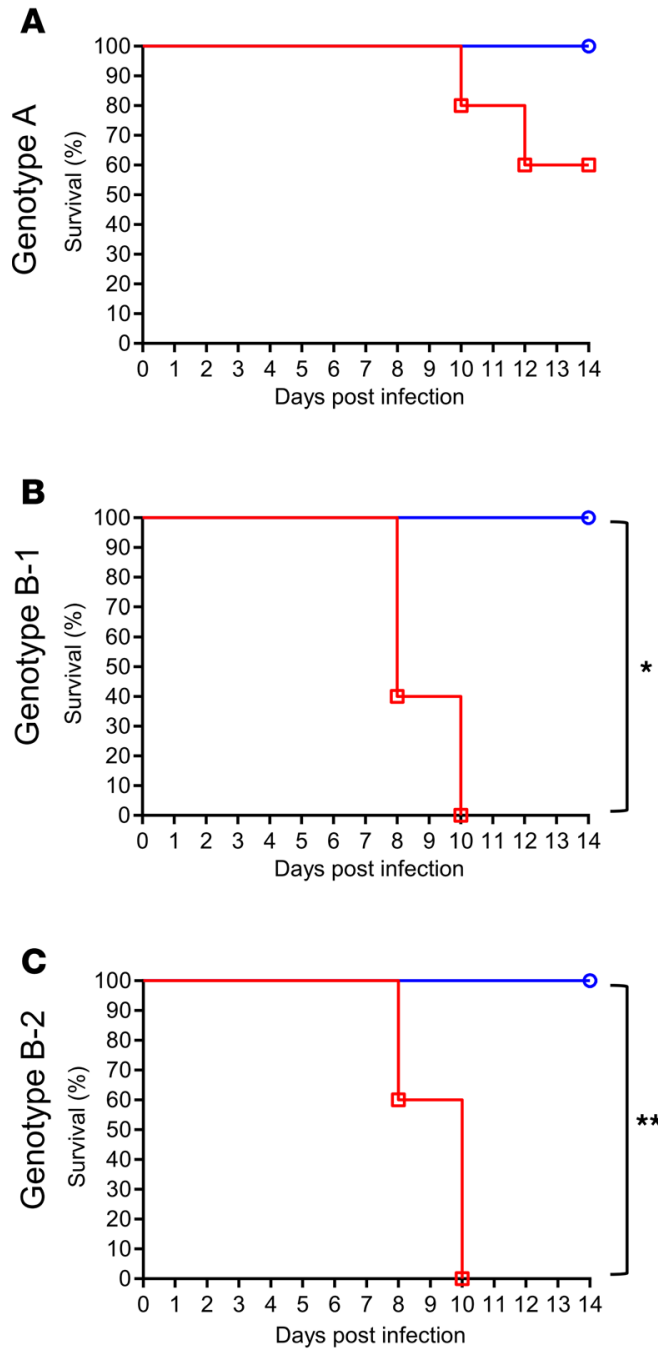

Viral load
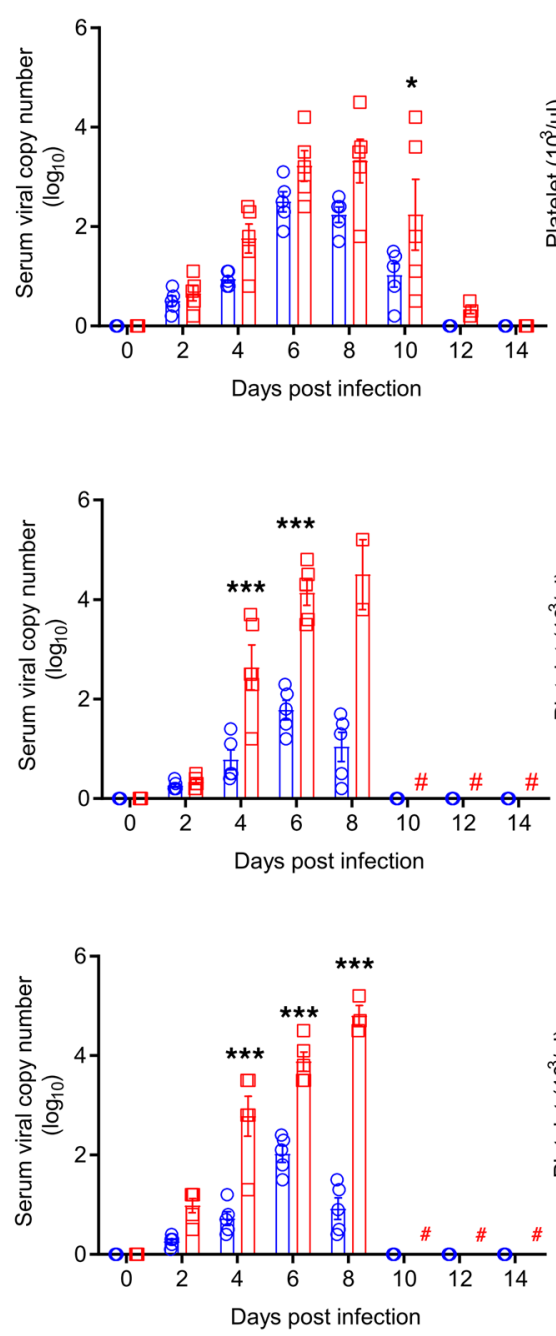

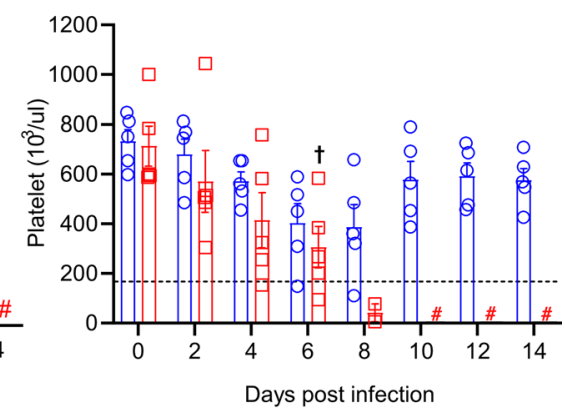

Platelet count
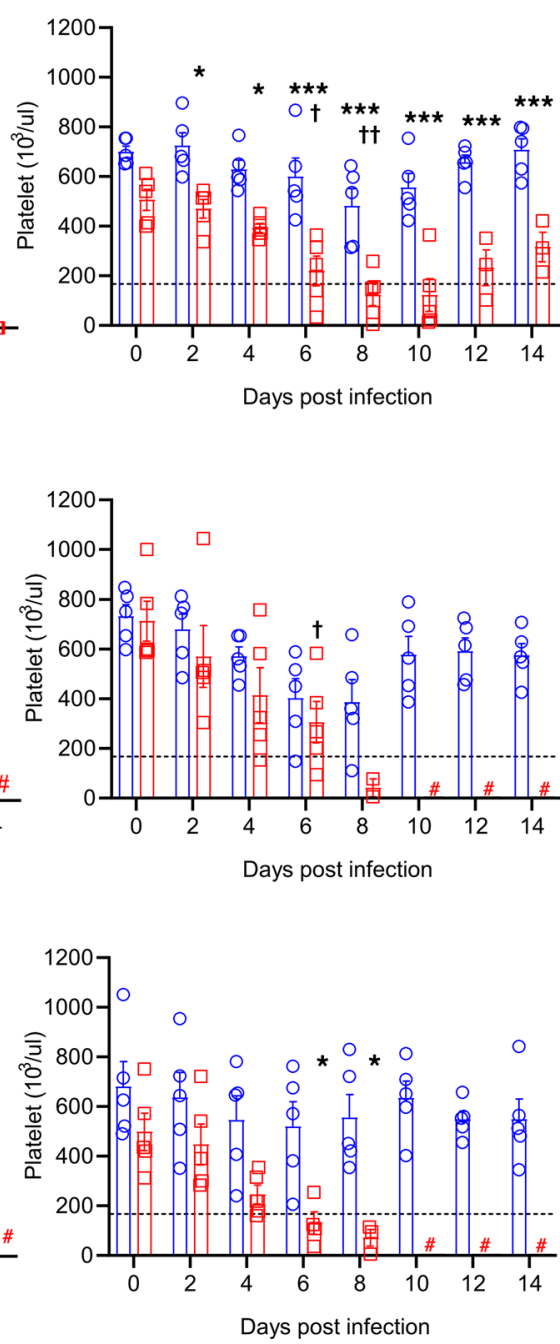

Figure 5. Pathogenicity of each randomly selected SFTSV pure genotype (A, B-1, B-2) in ferrets. Young adult ( $<2$ years, $n=5$; blue) or aged female and male ferrets ( $>4$ years, $n=5$; red) were inoculated with $10^{5.0} \mathrm{FFU} / \mathrm{mL}$ of each randomly selected SFTSV strain (A) A, (B) B-1, and (C) B-2. Survival, viral titers in serum, and platelet concentration were assessed. The normal range of platelets $\left(171.7 \times 10^{3} / \mu \mathrm{L}\right.$ to $\left.1280.6 \times 10^{3} / \mu \mathrm{L}\right)$ is marked by dashed lines in each panel. Both viral titers and platelet concentrations are presented as mean \pm SEM. Pound sign (\#) indicates no sample due to death of all ferrets of this group. Asterisks $\left({ }^{*}\right)$ indicate statistically significant difference between infected young adult and infected aged ferrets per dpi as determined by the Mantel-Cox method (survival) or 2-way ANOVA with Šidák's comparison (viral copy number and platelets). Crosses ( $\dagger$ ) indicate significant difference in platelet count between noninfected aged adult and infected aged ferret as calculated by 2 -way ANOVA with Tukey's test. ${ }^{*} P<0.05, \uparrow P<0.05, \dagger+P<0.001,{ }^{* * *} P<0.0001$.

\section{Discussion}

Due to the increasing incidence of human SFTSV infections, there is an elevated level of public concern. Therefore, in this study we investigated the epidemiological and genetic diversity of SFTSV strains in South Korea. In addition, we evaluated the pathogenic characteristics of each genotype of SFTSV using an experimental, age-dependent ferret model.

During the study period, a total of 3137 suspected SFTSV specimens were collected from 207 hospitals throughout South Korea, and 342 cases, mainly elderly, were confirmed as positive for SFTSV infection. Epidemiological investigation of SFTS cases in which virus was isolated showed that $38(32.8 \%)$ cases were fatal, and of these a majority of the patients were at least 50 years of age. The finding of high prevalence and mortality rate in the elderly is in agreement with corresponding data from other studies (30). The phy- 
Young adult ferrets

Aged ferrets

Platelet count
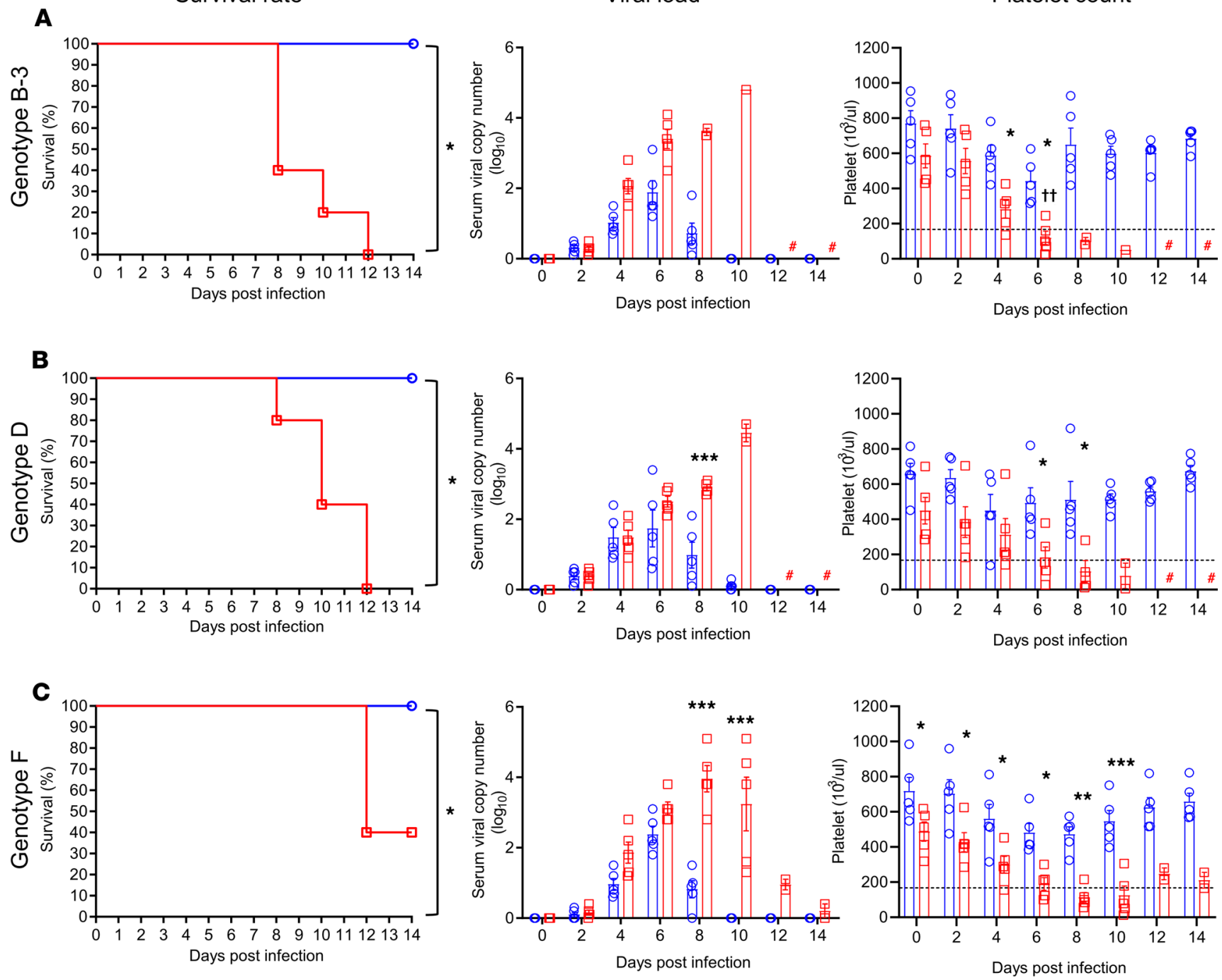

Figure 6. Pathogenicity of each randomly selected SFTSV pure genotype (B-3, D, F) in ferrets. Young adult ( $<2$ years, $n=5$; blue) or aged female and male ferrets ( $>4$ years, $n=5$; red) were inoculated with $10^{5.0} \mathrm{FFU} / \mathrm{mL}$ of each randomly selected SFTSV strain (A) B-3, (B) D, or (C) F. Survival, viral titers in serum, and platelet concentration were assessed. The normal range of platelets $\left(171.7 \times 10^{3} / \mu \mathrm{L}\right.$ to $\left.1280.6 \times 10^{3} / \mu \mathrm{L}\right)$ is marked by dashed lines in each panel. Both viral titers and platelet concentrations are presented as mean \pm SEM. Pound sign (\#) indicates no sample due to death of all ferrets of this group. Asterisks ${ }^{*}$ ) indicate statistically significant difference between infected young adult and aged infected ferrets per dpi as determined by Mantel-Cox method (survival) or 2-way ANOVA with Šidák's comparison (viral copy number and platelets). Crosses ( $\dagger$ ) indicate significant difference in platelet count between noninfected aged adult and infected aged ferrets as calculated by 2-way ANOVA with Tukey's test. ${ }^{*} P<0.05$ and ${ }^{* *} P<0.001, \dagger \dagger P<0.001,{ }^{* * *} P<0.0001$

logenetic tree of 335 SFTSV strains, including 116 strains identified in this study, revealed that most Korean SFTSVs can be classified into the 6 previously identified genotypes $(\mathrm{A}-\mathrm{F})(19,26)$, and the B genotype can be subdivided into at least 3 different genotypes (B-1, B-2, and B-3). Further, inconsistent phylogenetic clustering segments suggested that at least 6 genotypes were co-circulating and 9 reassortments among them in South Korea. The segmented nature of the Banyangvirus lends itself to genetic reassortment, which is an important molecular mechanism contributing to the genetic diversity necessary for viral evolution (31). Although there have been a few reports of genetic reassortment of SFTSV in China and Japan $(21,31,32)$, this is the first evidence to our knowledge of large and complex reassortment events, as at least 9 different reassortant genotypes were present in South Korea (Figure 3B). This suggests that SFTSV strains undergo evolution in nature through reassortment, resulting in the creation of novel genotypes. 
Genetic and phylogenetic analyses revealed that the B-2 $(n=48,36.1 \%)$ genotype was the most common in South Korea, followed by B-3 $(n=28,21.1 \%)$ and B-1 $(n=16,12.0 \%)$ (Figure 3A), while the F $(n=9)$ and $\mathrm{D}(n=5)$ genotypes were infrequently found. The highest mortality and incidence rates were observed in patients infected with genotype B-2 (43.8\%, 21 of 48 cases). In contrast, of 10 patients with genotype A, only a single case was fatal (10\%). The reported average case fatality rate associated with SFTSV infections varied greatly in East Asia, with China at 5.3\%-16.2\%, Japan at 20\%, and South Korea at $23.3 \%(11,33,34)$. Therefore, comparison of the virus genotype and mortality rates suggests that the differences in reported case mortality rates might be associated with the differential distribution of SFTSV genotypes across countries. Interestingly, genetic analysis of reported full-length sequences revealed that the most prevalent genotype in Japan was B-2, which exhibited a high case mortality rate, while genotypes $\mathrm{A}$ and $\mathrm{F}$ were not reported. In contrast, the most prevalent genotype in China was genotype F (44.3\%), followed by genotype A (21.5\%), both of which exhibited relatively low case mortality rates in Korea (35). These results suggest that, in addition to the age of the patients, prevalence of different genotypes in each country might also be a factor in the varied mortality rates between countries.

Previous studies demonstrated that aged ferrets mimic clinical signs (fever, hematological changes) of human SFTSV infection (27-29). Further, severe illness was exhibited only in aged ferrets, which is similar to that seen in humans older than 60 years. Thus, these results demonstrate that aged ferrets are a suitable SFTSV animal model. Therefore, we used aged ferrets to investigate genotype-dependent differences in the pathogenesis of SFTSV infection. Aged ferrets infected with genotypes B (B-1, B-2, and B-3), D, R-2, R-3, and R-5 showed $100 \%$ mortality, while genotypes A, F, R-1, R-4, R-6, and R-7 induced attenuated virulence with attenuated viral titers and delayed death, with $40 \%$ to $60 \%$ mortality, respectively (Figure 5, Figure 6, and Supplemental Figure 5). Further, only 1 of 5 aged ferrets infected with genotypes R-8 and R-9 died from the infection, and these groups of ferrets exhibited only moderate body weight loss (Supplemental Figure 4, H and I). These results are similar to what was reported for genotypic variants of Rift Valley fever viruses, which exhibited different levels of virulence in a mouse model (36). However, the low number of clinical cases of infection with certain SFTSV genotypes, especially of the reassortant genotypes, impedes the determination of the association between genotype and case mortality. Further, recently, Song et al. demonstrated that fatality induced by SFTSV infection is associated with the absence of specific IgGs to viral nucleocapsid and glycoprotein due to a failure in B cell class switching (37). Similarly, in our preliminary ELISA study with ferret sera, we found that SFTSV-infected healthy young ferrets exhibited a strong IgG response, while aged ferrets showed very limited IgG responses against the NP of the B-1/2014 SFTSV strain (unpublished observations). However, in contrast to human cases, most fatalities occurred in 10-12 days after infection of ferrets, which is a relatively short time to induce full antibody responses against antigens. Therefore, it is hard to determine if there is an association between the absence of virus-specific B cell immunity and mortality in this study. In this regard, further continuous surveillance of SFTS patients with genomic and pathogenic analyses of detailed clinical manifestations are needed to better understand the association between fatality and specific genotypes.

Overall, the evidence from this study increases our understanding of the genetic and pathogenic diversity of SFTSVs in East Asian countries. Virological and clinical analyses revealed that there are close associations among the clinical manifestations (case fatality), patient age, and SFTSV genotype. In addition, continuous surveillance studies are needed to contribute to beneficial for the preventions of severe outbreaks of SFTSV infections.

\section{Methods}

Specimen collection from patients with suspected SFTS and clinical evaluation. A total of 3137 specimens (sera or cerebrospinal fluid) were collected from SFTS-suspected patients who experienced symptoms of SFTS, such as high fever $\left(\geq 38^{\circ} \mathrm{C}\right)$, vomiting, diarrhea, fatigue, thrombocytopenia, and leukocytopenia in 207 hospitals throughout South Korea between 2013 and April 2017. SFTSV infection was confirmed in collected specimens by one-step RT-PCR using an $M$ segment-based SFTSV-specific primer set (MF3 [5'-GATGAGATGGTCCATGCTGATTCT-3'] and MR2 [5'-CTCATGGGGTGGAATGTCCTCAC-3']) (38). To compare demographic characteristics of SFTS cases, information regarding age, sex, occupation, residential address, and presence or absence of death was also collected in an epidemiologic investigation of SFTS patients.

SFTSV isolation from patient specimens. For virus isolation, Vero E6 cells (derived from an African green monkey kidney epithelial cell [CRL-1586; ATCC]) were inoculated with the patient specimens and cultured in DMEM (Gibco) containing 2\% FBS (Gibco) with penicillin and streptomycin (P/S, Gibco) and 
placed in a $37^{\circ} \mathrm{C}$ incubator supplemented with $5 \% \mathrm{CO}_{2}$. After 14 days of incubation, isolated viruses were identified with RT-PCR. To make the stock viruses but minimize the unnecessary mutation of SFTSV during the virus isolation, 1 or 2 addition cell cultures were conducted and stored at $-80^{\circ} \mathrm{C}$ until further use.

Genetic characterization and phylogenetic analysis. For whole genome sequencing, PCR fragments covering whole SFTSV genome were amplified by RT-PCR. Whole SFTSV genome cDNA libraries for use in deep sequencing using the next-generation sequencing (NGS) method were prepared using the Nextera XT DNA Library Preparation Kit (Illumina) with an average insert size of $300 \mathrm{bp}$. The sequencing run was performed on a MiniSeq system (Illumina) using a MiniSeq mid-output kit, resulting in $2 \times 150$ nucleotides paired-end reads (39). PCR primer sets (5' to 3') are L1F: ACACAGAGACGCCCAGATGGACT, L1308F: AGAAGTGGAAGAGAATGGCAG, L2261F: AGCTGGTACATTGGGTACTACAAGAA, L3518F: TCAGAGAAATCAACAGTGAACAC, L4697F: TTTCTTAGCCATGTGCAGTTTAG, L1638R: AACTCACCAGCCCTGCAGTG, L2748R: AAGAGACAGATTCGCATGCACCC, L3762R: AGCATGTAGTGGTGCAGGAGCTG, L4995R: GGTATCACAAGAGTAGAGAAGGC, L6268R: ACACAAAGACCGCCCAGATCTTAA, M1F: ACACAGAGACGGCCAACAATGA, M1492F: TTTGCCATCATAAAGAAACT, M1673R: AGGATGACAGGTGAGTACATCC, M3378R: ACACAAAGACCGGCCAACACTTCA, S1F: ACACAAAGAACCCCCTTCATT, S1029F: TGGGCCAAGGATTCCCTTGGCCT, S1424R: GAGAGGGCAGAAACCAGGCTCTC, S1747R: ACACAAAGAACCCCCAAAAAA. The nucleotide sequences obtained from this study were assembled using the QIAGEN Bioinformatics CLC Workbench program (version 10.1.1; CLC bio).

Genetic and phylogenetic analyses were conducted by aligning published full-length sequences of SFTSV obtained from China, Japan, and South Korea (GenBank). A total of 335 full-length sequences of the $L, M$, and $S$ segments (158 from China, 43 from Japan, and 134 from South Korea including this study) were included in the analyses (Supplemental Table 4). Multiple sequence alignments were performed using the ClustalW algorithm in MEGA version 6.0 (40). Phylogenetic analyses were performed based on the complete ORF sequences of $L, M$, and $S$ segments of SFTSVs using the maximum likelihood (ML) method based on Kimura's 2-parameter model. The reliability of the ML tree was evaluated by the bootstrap test with 1000 replications.

In vitro growth properties of each virus genotype in cells. Vero E6 cells were cultured in 6-well plates and then infected with each virus genotype at an MOI of 0.001 for 1 hour, washed and incubated at $37^{\circ} \mathrm{C}$ in a $5 \% \mathrm{CO}_{2}$ atmosphere. Cell culture supernatants were collected at 1-6 dpi, and virus titers were determined by FFU $/ \mathrm{mL}$.

Ferret infection studies with different SFTSV genotypes. Of the isolated SFTSV strains, a total of 15 different genotypes of SFTSVs were selected and inoculated into each group of young adult ( $<2$ years old, Mustela putorius furo) or aged female and male ferrets ( $>4$ years old, Mustela putorius furo) $(n=5)$ (ID bio), respectively, with $10^{5.0} \mathrm{FFU} / \mathrm{mL}$ strain as previously described (27). For the analysis of hematological parameters and viral load, blood was collected every other day for 14 days in EDTA tubes and analyzed for hematological parameters using a Celltac hematology analyzer (MEK-6550J/K, Nihon Kohden). For virus titration in sera from infected animals, the number of viral RNA copies was measured as previously described (27).

Study approval. Viruses were isolated from serum samples collected from Chungbuk National University Hospital, in accordance with procedures approved by the Institutional Review Board of Chungbuk National University Hospital (IRB 2017-05-002-001) and Korea Centers for Disease Control and Prevention (KCDC). The KCDC is legally tasked with the laboratory diagnosis and related research using the remaining serum samples for SFTSV patients upon request by medical institutions in accordance with the Act on the Prevention and Control of Infectious Diseases, Thus, informed consent was waived by the institutional review board (IRB) of the KCDC (IRB no. 2014-01CON-06-P-A). All animal experiments were approved by the Medical Research Institute, a member of the Laboratory Animal Research Center of Chungbuk National University (LARC) (approval CBNUA-1804-18-01), and were conducted in strict accordance with and adherence to relevant policies as mandated under the Guidelines for Animal Use and Care of the Korea Centers for Disease Control (K-CDC) in an enhanced biosafety level 3 (BSL3) containment laboratory.

Statistics. Asterisks indicate statistically significant differences in mortality rates between infected patient groups according to their SFTSV genotype; and in weight loss, temperature change, blood analysis, viral load, and titer between groups and across time points as determined by 2-tailed Mantel-Cox method, Mann-Whitney U test, 2-way ANOVA with Šidák's comparison, or 2 way ANOVA with Tukey's test. Statistical analyses were performed using GraphPad Prism version 8.2.0 for Windows (GraphPad Software). $P$ values less than 0.05 were considered statistically significant. 


\section{Author contributions}

SMY, SJP, YIK, and YKC conceived and designed the study. SMY, SJP, YIK, SWP, MAY, HIK, EHK, KMY, HWJ, JR, WJL, YJ, and JYL conducted sample collection and virus isolation. SMY, SJP, YIK, and HWJ analyzed sample sequences and data. SMY, SJP, YIK, JYL, and YKC wrote the manuscript.

\section{Acknowledgments}

We thank all involved staff members in the diagnosis of SFTS and epidemiological investigation of SFTS patients during 2013-2017. This work was supported by the Korean Ministry of Health and Welfare (government-wide R\&D fund project for infectious disease research [HG18C0029]) and supported by the intramural fund (2017-NI53002-00) of the Research of National Institute of Health, Korea Centers for Disease Control and Prevention.

Address correspondence to: Joo-Yeon Lee, Division of Emerging Infectious Disease and Vector Research, Center for Infectious Diseases Research, National Institute of Health, Korea Centers for Disease Control and Prevention, 187 Osongsaengmyeong 2-ro, Osong-eup, Cheongju, Chungcheongbuk-do, 28159, South Korea. Phone: 82.43.719.8490; Email: 1jyljy@nih.go.kr. Or to: Young Ki Choi, College of Medicine and Medical Research Institute, Chungbuk National University, 1 Chungdae-ro, Seowon-gu, Cheongju, Chungcheongbuk-do, 28644, South Korea. Phone: 82.43.261.3384; Email: choiki55@chungbuk.ac.kr.

1. Moore JR, Schneider SM. Acute human immunodeficiency virus (HIV) infection presenting with fever, elevated amylase/lipase, and hematologic abnormalities. J Emerg Med. 2013;44(5):e341-e344.

2. Yu XJ, et al. Fever with thrombocytopenia associated with a novel bunyavirus in China. N Engl J Med. 2011;364(16):1523-1532

3. Takahashi T, et al. The first identification and retrospective study of severe fever with thrombocytopenia syndrome in Japan. $J$ Infect Dis. 2014;209(6):816-827.

4. Kim KH, et al. Severe fever with thrombocytopenia syndrome, South Korea, 2012. Emerging Infect Dis. 2013;19(11):1892-1894

5. Abudurexiti A, et al. Taxonomy of the order Bunyavirales: update 2019. Arch Virol. 2019;164(7):1949-1965.

6. Maes P, et al. Taxonomy of the order Bunyavirales: second update 2018. Arch Virol. 2019;164(3):927-941

7. Shen S, et al. A novel tick-borne phlebovirus, closely related to severe fever with thrombocytopenia syndrome virus and Heartland virus, is a potential pathogen. Emerg Microbes Infect. 2018;7(1):95.

8. McMullan LK, et al. A new phlebovirus associated with severe febrile illness in Missouri. N Engl J Med. 2012;367(9):834-841.

9. Liu Q, He B, Huang SY, Wei F, Zhu XQ. Severe fever with thrombocytopenia syndrome, an emerging tick-borne zoonosis. Lancet Infect Dis. 2014;14(8):763-772.

10. [No authors listed]. KCDC Infectious Disease Portal Web Site. http://www.cdc.go.kr/npt/biz/npp/ist/simple/simplePdStatsMain.do. Accessed January 8, 2020.

11. Zhan J, et al. Current status of severe fever with thrombocytopenia syndrome in China. Virol Sin. 2017;32(1):51-62.

12. [No authors listed]. Severe fever with thrombocytopenia syndrome (SFTS) in Japan, as of February 2016. IASR 37. 2016;37(3):39-40.

13. Park SW, Han MG, Yun SM, Park C, Lee WJ, Ryou J. Severe fever with thrombocytopenia syndrome virus, South Korea, 2013. Emerging Infect Dis. 2014;20(11):1880-1882.

14. Kitao A, et al. Severe fever with thrombocytopenia syndrome presenting as hemophagocytic syndrome: two case reports. Spring erplus. 2016;5:361.

15. Li DX. Severe fever with thrombocytopenia syndrome: a newly discovered emerging infectious disease. Clin Microbiol Infect. 2015;21(7):614-620

16. Deng B, et al. Clinical features and factors associated with severity and fatality among patients with severe fever with thrombocytopenia syndrome Bunyavirus infection in Northeast China. PLoS One. 2013;8(11):e80802.

17. Sun J, et al. Factors associated with severe fever with thrombocytopenia syndrome infection and fatal outcome. Sci Rep. 2016;6:33175.

18. Liu MM, Lei XY, Yu XJ. Meta-analysis of the clinical and laboratory parameters of SFTS patients in China. Virol J. 2016;13(1):198.

19. Fu Y, et al. Phylogeographic analysis of severe fever with thrombocytopenia syndrome virus from Zhoushan Islands, China: implication for transmission across the ocean. Sci Rep. 2016;6:19563.

20. Yoshikawa T, et al. Phylogenetic and geographic relationships of severe fever with thrombocytopenia syndrome virus in China, South Korea, and Japan. J Infect Dis. 2015;212(6):889-898.

21. Liu JW, et al. Molecular evolution and spatial transmission of severe fever with thrombocytopenia syndrome virus based on complete genome sequences. PLoS One. 2016;11(3):e0151677.

22. Song $P$, et al. Downregulation of interferon- $\beta$ and inhibition of tlr3 expression are associated with fatal outcome of severe fever with thrombocytopenia syndrome. Sci Rep. 2017;7(1):6532.

23. Liu J, Wang L, Feng Z, Geng D, Sun Y, Yuan G. Dynamic changes of laboratory parameters and peripheral blood lymphocyte subsets in severe fever with thrombocytopenia syndrome patients. Int J Infect Dis. 2017;58:45-51.

24. Deng B, et al. Cytokine and chemokine levels in patients with severe fever with thrombocytopenia syndrome virus. PLoS One. 2012;7(7):e41365. 
25. Park SY, et al. Severe fever with thrombocytopenia syndrome-associated encephalopathy/encephalitis. Clin Microbiol Infect. 2018;24(4):432.e1-432.e4

26. Yun SM, et al. Molecular genomic characterization of tick- and human-derived severe fever with thrombocytopenia syndrome virus isolates from South Korea. PLoS Negl Trop Dis. 2017;11(9):e0005893.

27. Park SJ, et al. Ferret animal model of severe fever with thrombocytopenia syndrome phlebovirus for human lethal infection and pathogenesis. Nat Microbiol. 2019;4(3):438-446.

28. Yu KM, et al. Shedding and transmission modes of severe fever with thrombocytopenia syndrome phlebovirus in a ferret model. Open Forum Infect Dis. 2019;6(8):ofz309.

29. Kwak JE, et al. Development of a SFTSV DNA vaccine that confers complete protection against lethal infection in ferrets. Nat Commun. 2019;10(1):3836

30. Sun J, Lu L, Wu H, Yang J, Ren J, Liu Q. The changing epidemiological characteristics of severe fever with thrombocytopenia syndrome in China, 2011-2016. Sci Rep. 2017;7(1):9236.

31. Shi J, et al. Migration, recombination, and reassortment are involved in the evolution of severe fever with thrombocytopenia syndrome bunyavirus. Infect Genet Evol. 2017;47:109-117.

32. Li Z, et al. Increased prevalence of severe fever with thrombocytopenia syndrome in Eastern China clustered with multiple genotypes and reasserted virus during 2010-2015. Sci Rep. 2017;7(1):6503.

33. Gokuden M, et al. Low seroprevalence of severe fever with thrombocytopenia syndrome virus antibodies in individuals living in an endemic area in Japan. Jpn J Infect Dis. 2018;71(3):225-228.

34. [No authors listed]. Severe fever thrombocytopenia syndrome (SFTS). Information of infectious diseases. Tokyo: National Institute of Infectious Diseases. https://www.niid.go.jp/niid/ja/diseases/sa/sfts.html. Accessed December 16, 2019.

35. Robles NJC, Han HJ, Park SJ, Choi YK. Epidemiology of severe fever and thrombocytopenia syndrome virus infection and the need for therapeutics for the prevention. Clin Exp Vaccine Res. 2018;7(1):43-50

36. Ikegami T, et al. Distinct virulence of Rift Valley fever phlebovirus strains from different genetic lineages in a mouse model. PLoS One. 2017;12(12):e0189250.

37. Song P, et al. Deficient humoral responses and disrupted B-cell immunity are associated with fatal SFTSV infection. Nat Commun. 2018;9(1):3328.

38. Yun SM, et al. Severe fever with thrombocytopenia syndrome virus in ticks collected from humans, South Korea, 2013. Emerging Infect Dis. 2014;20(8):1358-1361.

39. [No authors listed]. An introduction to next-generation sequencing technology. Illumina. https://www.illumina.com/documents/products/illumina_sequencing_introduction.pdf. Accessed December 16, 2019

40. Tamura K, Stecher G, Peterson D, Filipski A, Kumar S. MEGA6: Molecular Evolutionary Genetics Analysis version 6.0. Mol Biol Evol. 2013;30(12):2725-2729. 